\title{
Antithrombotic treatment patterns in patients with atrial fibrillation in Italy pre- and post-DOACs: the REPAIR study
}

\author{
Luca D Esposti*,1, Jean-Baptiste Briere², Kevin Bowrin², Sangiorgi Diego1, \\ Valentina Perrone ${ }^{1}$ \& Giuseppe Di Pasquale ${ }^{3}$ \\ ${ }^{1} \mathrm{CliCon} \mathrm{Srl}$, Health Economics and Outcomes Research, Ravenna, Italy \\ ${ }^{2}$ Bayer AG, Berlin, Germany \\ ${ }^{3}$ Dipartimento Medico Azienda USL di Bologna. Unità Operativa di Cardiologia Ospedale Maggiore, Bologna, Italy \\ *Author for correspondence: Tel.: +39 544 38393; Fax: +39 544212 699; luca.degliesposti@clicon.it
}

\begin{abstract}
Aim: To evaluate antithrombotic treatment patterns in patients in Italy with nonvalvular atrial fibrillation (NVAF) before and after direct oral anticoagulants (DOACs) were approved. Methods: This analysis included patients with a discharge diagnosis of NVAF in 2010 and 2014, which constituted the pre- and post-DOACs populations, respectively. Results: Approximately $90 \%$ of patients were eligible for oral anticoagulant (OAC) therapy. Overall use of OACs increased from $38 \%$ in 2010 to $45 \%$ in 2014; use of antiplatelet therapy decreased from 36 to $25 \%$. Approximately $14 \%$ of eligible patients remained untreated. Conclusion: Although an improvement in OAC prescription was observed post-DOACs launch, treatment patterns in Italy suggest that a proportion of patients with NVAF are still undertreated or do not receive appropriate therapy.
\end{abstract}

First draft submitted: 19 January 2018; Accepted for publication: 30 November 2018; Published online: 21 January 2019

Keywords: antithrombotic treatment patterns $\bullet$ direct oral anticoagulants $\bullet$ nonvalvular atrial fibrillation $\bullet$ OAC prescription • real-world data

Atrial fibrillation (AF) affects approximately $2 \%$ of the Italian population $[1,2]$, which is within the $1-2 \%$ range of the whole European population [3]. Nonvalvular AF (NVAF) is the most common sustained cardiac arrhythmia encountered in clinical practice. NVAF increases the risk of thromboembolic events, particularly the risk of ischemic stroke by fivefold and leads to $15-20 \%$ of all ischemic strokes [4]. Antithrombotic prophylaxis to prevent thromboembolism is recommended for all patients with NVAF, except in those who are at low risk (aged $<65$ years and lone AF), or with contraindications. In particular, the 2016 European Society of Cardiology (ESC) guidelines recommend that in patients with a $\mathrm{CHA}_{2} \mathrm{DS}_{2}$-VASc score $\geq 2$ oral anticoagulant $(\mathrm{OAC})$ treatment is mandatory (class IA) [5].

Direct oral anticoagulants (DOACs) such as apixaban, dabigatran, edoxaban or rivaroxaban were launched in Europe several years ago, with the aim of offering patients with NVAF, an efficient and alternative treatment to vitamin K antagonists (VKAs) for the prevention of ischemic stroke or systemic embolism [6]. Several Global and European registries have demonstrated an overall increase in anticoagulant use and adherence to guidelines for patients with NVAF since the introduction of DOACs [7-10], albeit other studies suggest that improvements are still needed [11-13]. Less is known about treatment patterns at the country level. Of the available data, Italy has been noted as a country with one of the lowest rates of DOAC utilization since their introduction in Europe [14]; however, data specific to Italy are only available up to 2013 when DOACs were first made reimbursable. This warranted a more recent analysis of DOAC use for patients with NVAF in Italy and presented an opportunity to better understand treatment patterns associated with non-adherence to guideline recommendations.

The aim of this retrospective cohort study was to evaluate treatment patterns in Italian patients with a discharge diagnosis of NVAF, who should receive anticoagulant therapy as per ESC guidelines, before and after the DOACs were approved. In addition, patients' characteristics (in terms of age and comorbidity index) and risks (based on $\mathrm{CHA}_{2} \mathrm{DS}_{2}$-VASc, HAS-BLED scores) were also assessed. 


\section{Methods}

\section{Data sources}

An observational retrospective cohort analysis based on administrative databases (DBs) of six Italian local health units (LHUs), geographically distributed throughout the country, was conducted (for a total number of 4 million health-assisted individuals).

The structure of this DB has been described in detail elsewhere [15]. It includes various DBs: beneficiaries' DB, containing patients' demographic data; pharmaceuticals prescription DB, providing information for each drug prescription, such as the anatomical therapeutic chemical (ATC) code; hospital discharge DB, containing information on the diagnosis-related group reimbursement rate, reasons for hospitalization according to the International Classification of Diseases, Ninth Revision, Clinical Modification (ICD-9-CM); outpatient specialist services DB, collecting information on visits or outpatient services provided to patients; and laboratory tests DB, containing blood test results for each patient. A unique anonymized patient identification program allowed linkage of data across all DBs. According to Italian regulations [16], informed consent is not required for anonymized retrospective information; however, local ethics committees of the participating LHUs approved the study.

\section{Cohort definition}

Patients with a discharge diagnosis of NVAF (ICD-9-CM code: 427.31) during 2010 and 2014 were included. We excluded all patients with malignancies (ICD-9-CM codes: 140.XX-199.XX), pulmonary embolism/deep vein thrombosis (DVT; ICD-9-CM codes: 415, 451, 453), valvular AF (ICD-9-CM codes: V42, V43, 394, 395, 396) and major orthopedic surgery (ICD-9-CM codes according to Farner et al.) [17]. In addition, patients who required anticoagulation for AF and patients who were transferred to another LHU during the follow-up period were also excluded from the study.

The inclusion date was used as the index date, patients enrolled in 2010 constituted the pre-DOACs cohort, while patients enrolled in 2014 constituted the post-DOACs cohort. Patients enrolled were followed up for all time available from the index date (follow-up period) and their clinical characteristics were investigated before to the index date for a minimum duration of 1 year (characterization period).

Comorbidities were measured by using the Charlson Comorbidity Index (CCI) [18] and identified through hospitalizations, in other words, peptic ulcer (ICD-9-CM codes: 531-534), hemorrhage/bleeding (ICD-9-CM codes: 456.0, 531.0, 531.2, 531.4, 531.6, 532.0, 532.2, 532.4, 532.6, 533.0, 533.2, 533.4, 533.6, 534.0, 534.2, 534.6, 569.3, 578.0, 578.9, 596.7, 599.7, 626.5, 626.6, 626.9, 627.0, 627.1, 623.8, 626.2, 784.7, 786.3, 459.0, 719.1, 423.0, 379.2, 852.2), chronic bronchitis (ICD-9-CM: 491), in particular obstructive chronic bronchitis (ICD-9CM code: 491.2), asthma (ICD-9-CM code: 493), gastroesophageal reflux (ICD-9-CM code: 530.81), disorders of the thyroid gland (ICD-9-CM codes: 240-246), dementia (ICD-9-CM code: 290), other cerebral degenerations (ICD-9-CM code: 331), sleep apnea (ICD-9-CM codes: 780.51, 780.53, 780.57) and bone fractures (ICD-9-CM codes: $805,806,808,820,824,812.0-812.5,813.4,813.5,807.0,807.1)$ during the characterization period. Both primary and secondary diagnoses collected in the DB were considered. When a diagnosis was not available, the prescriptions of specific drugs was used as a proxy for the specific comorbidity, in other words, prescriptions due to the use of antihypertensive drugs (ATC codes: C02, C03, C07, C08, C09), statins (ATC code: C10AA), organic nitrates (ATC code: C01DA), antidiabetic drugs (ATC codes: A10A, A10B), anti-inflammatory and antirheumatic agents, non-steroids (ATC code: M01A), macrolides (ATC code: J01FA), proton pump inhibitors (ATC code: A02BC). The patients' stroke and bleeding risk were calculated using $\mathrm{CHA}_{2} \mathrm{DS}_{2}$-VASc and HAS-BLED scores, respectively.

Baseline characteristics were assessed for the overall population, as well as for the subgroup of patients with a $\mathrm{CHA}_{2} \mathrm{DS}_{2}$-VASc score of 2 or more, for whom the use of OACs is recommended by ESC guidelines [5]. For the subgroup analysis, patients were classified into five groups according to their antithrombotic therapies posthospital discharge: VKA, DOAC, other anticoagulant treatment, antiplatelet and no treatment. According to the therapeutic strategy used at the index date, the pharmaco-utilization profiles (in terms of switch rate and interruption) of patients were estimated during the follow-up period.

In order to have 1 year of follow-up analysis for all patients, we enrolled patients from January to June (data availability until June 2015); moreover, patients who died at or before 1 year were excluded. Therapeutic variations were assessed within the last 3 months of the follow-up period. In particular, we evaluated: the percentage of patients who switched their index therapy and the percentage of patients who interrupted any treatment prescribed during the follow-up period. 


\begin{tabular}{|c|c|c|c|}
\hline Baseline characteristics & Pre-DOACs & Post-DOACs & p-value \\
\hline Age, mean \pm SD & $74.8 \pm 11.7$ & $76.6 \pm 11.4$ & $<0.001$ \\
\hline Male, N (\%) & 3659 (49) & $3493(49)$ & 0.462 \\
\hline Charlson Comorbidity Index, mean \pm SD & $1.5 \pm 1.5$ & $1.6 \pm 1.4$ & 1.000 \\
\hline $\begin{array}{l}\text { Comorbidities of the hepatobiliary } \\
\text { system and pancreas, N (\%) }\end{array}$ & $147(2.0)$ & $105(1.5)$ & 0.020 \\
\hline $\mathrm{CHA}_{2} \mathrm{DS}_{2}$-VASc score, mean $\pm \mathrm{SD}$ & $3.5 \pm 1.6$ & $3.7 \pm 1.5$ & $<0.001$ \\
\hline HAS-BLED score, mean $\pm S D$ & $2.3 \pm 1.1$ & $2.5 \pm 1.0$ & $<0.001$ \\
\hline
\end{tabular}

DOAC: Direct oral anticoagulant; SD: Standard deviation.

Table 2. Demographic and baseline clinical characteristics of patients with nonvalvular atrial fibrillation enrolled in 2010 and 2014 and eligible for oral anticoagulants - (CHA DS $_{2}$-VASc score $\left.\geq 2\right)$.

\begin{tabular}{|llll}
\hline N & Pre-DOACs & Post-DOACs & p-value \\
\hline Age, mean \pm SD & 6578 & 6602 & $78.4 \pm 9.4$ \\
\hline Male, N (\%) & $77.2 \pm 9.4$ & $2990(45)$ & 0.471 \\
\hline Charlson Comorbidity Index, mean \pm SD & $1.6 \pm 1.5$ & $1.7 \pm 1.4$ & $154(2.3)$ \\
\hline $\begin{array}{l}\text { Comorbidities of the kidney and urinary } \\
\text { tract, N (\%) }\end{array}$ & $132(2.0)$ & $104(1.6)$ & 0.001 \\
\hline $\begin{array}{l}\text { Comorbidities of the hepatobiliary } \\
\text { system and pancreas, N (\%) }\end{array}$ & $144(2.2)$ & $3.9 \pm 1.2$ & 0.011 \\
\hline CHA 2 DS - VASc score, mean \pm SD & $3.8 \pm 1.3$ & $2.6 \pm 0.9$ \\
\hline HAS-BLED score, mean \pm SD & $2.5 \pm 1.0$ & $<0.001$ \\
\hline DOAC: Direct oral anticoagulant; SD: Standard deviation. & $<0.001$ \\
\hline
\end{tabular}

The index Major Diagnostic Categories (MDCs) and the length of the index hospitalization stay of patients with NVAF enrolled in 2010 and 2014 and eligible for OACs - $\left(\mathrm{CHA}_{2} \mathrm{DS}_{2}\right.$-VASc score $\left.\geq 2\right)$ were also evaluated.

\section{Statistical analysis}

Continuous variables were reported as mean and standard deviation $( \pm S D)$, whereas categorical variables were expressed as numbers and percentages. The p-values $\leq 0.05$ were considered to be statistically significant. All statistical analyses were performed using Stata software version 12.0 (Stata Corp LP, TX, USA), and data management was carried out using Microsoft SQL Server 2012.

\section{Results}

After applying inclusion and exclusion criteria, 7416 patients with a discharge diagnosis of NVAF were enrolled (pre-DOACs cohort) in 2010, compared with 7170 patients in 2014 (post-DOACs cohort). Mean age was 74.8 years in 2010 and 76.6 years in 2014, CCI for comorbidities remained similar in the two cohorts (1.5 and 1.6, respectively). Average $\mathrm{CHA}_{2} \mathrm{DS}_{2}$-VASc score was 3.5 in the pre-DOACs cohort and 3.7 in the post-DOACs cohort, HAS-BLED scores were 2.3 and 2.5 , respectively (Table 1 ).

Baseline characteristics among patients with NVAF were assessed, as well as for the subgroup of patients with a $\mathrm{CHA}_{2} \mathrm{DS}_{2}$-VASc score of 2 or more, for whom the use of OACs is recommended by the ESC (Table 2).

In 2010, 6578 (89\% of the original cohort) patients with a discharge diagnosis of NVAF and 6602 ( $92 \%$ of the original cohort) in 2014 were eligible for OACs as per ESC guidelines. Mean age was 77.2 years in the pre-DOACs cohort and 78.4 years in the post-DOACs cohort, $\mathrm{CHA}_{2} \mathrm{DS}_{2}$-VASc score was 3.8 in 2010 and 3.9 in 2014 , respectively, HAS-BLED values were 2.5 and 2.6 in 2010 and 2014, respectively.

Treatment patterns were assessed in both cohorts at enrolment, results are shown in Figure 1. In the pre-DOACs cohort, $38 \%$ of patients were treated with a VKA, 36\% with an antiplatelet, $12 \%$ with other anticoagulant 

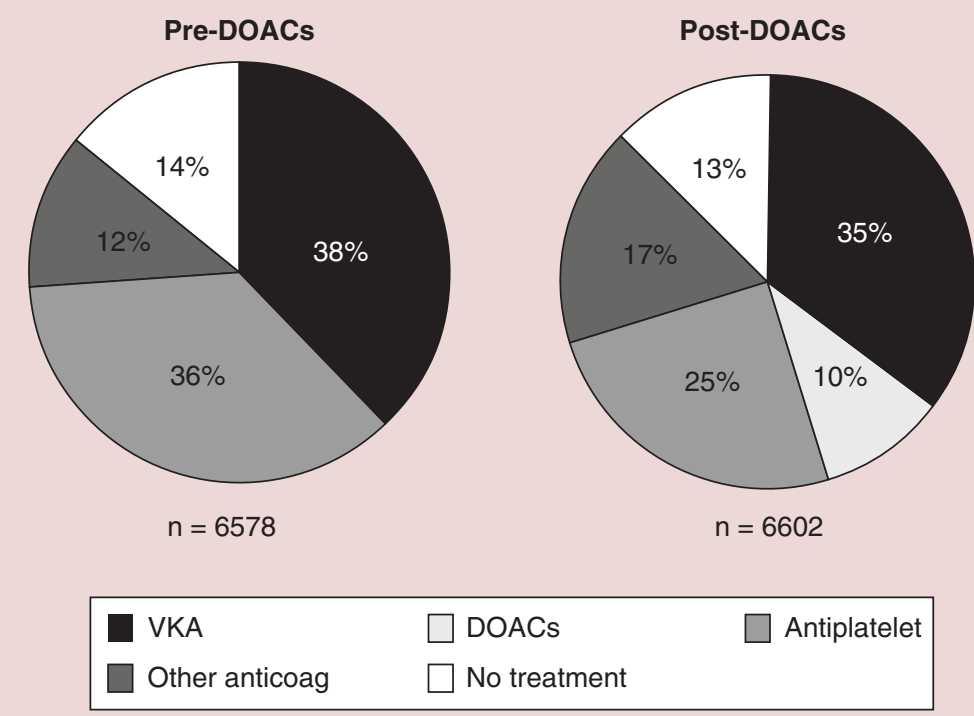

Figure 1. Treatment patterns in 2010 and 2014 in patients eligible for oral anticoagulants $-\mathrm{CHA}_{2} \mathrm{DS} \mathrm{S}_{2}$-VASC Score $\geq 2$. DOAC: Direct oral anticoagulant; Other anticoag: Other anticoagulant treatment; VKA: Vitamin K antagonist.

agents (such as low molecular weight heparin) and the remaining 14\% received no treatment. In the post-DOACs cohort, $35 \%$ of patients were treated with a VKA, $10 \%$ with a DOAC, $25 \%$ with an antiplatelet, $17 \%$ with other anticoagulant agents and $13 \%$ received no treatment.

Demographic and baseline clinical characteristics of patients with NVAF enrolled in 2010 and 2014 and eligible for OACs were explored according to their treatment at the index date (Table 3).

In the pre-DOACs cohort, mean age ranged from 74.9 years in patients treated with VKA to 79.5 years in patients not treated with antithrombotic agents. $\mathrm{CHA}_{2} \mathrm{DS}_{2}$-VASc scores were 3.8, 3.9, 4.0 and 3.5 in patients treated with VKA, antiplatelet, other anticoagulant agents or without any antithrombotic treatment, respectively; HAS-BLED scores were 2.4, 2.6, 2.7 and 2.0, respectively. In the post-DOACs cohort, mean age ranged from 76.6 years in patients treated with VKA to 80.2 years in patients treated with an antiplatelet, mean age was 77.5 years in patients treated with DOACs, 79.2 years in patients treated with other anticoagulant agents and 79.7 years in NVAF patients without any antithrombotic agent prescribed. $\mathrm{CHA}_{2} \mathrm{DS}_{2}$-VASc scores were similar across the groups: 3.8 in VKA-treated patients, 3.9 in the DOAC-treated group, 4.1 in the antiplatelet-treated group, 4.0 for patients treated with other anticoagulant agents and 3.8 in patients without antithrombotic treatment. Furthermore, HAS-BLED values were also similar: 2.6 for DOAC-treated patients, 2.8 for antiplatelet-treated patients, 2.7 for patients treated with other anticoagulant, 2.5 in patients treated with VKA and 2.5 in patients not treated with antithrombotics. Statistical analysis of pre- versus post-DOACs treatment patterns demonstrated that patients who received a VKA in the post- compared with pre-DOAC era were older with a higher CCI for comorbidities and HAS-BLED score (all $\mathrm{p}<0.01$ ). Patients receiving other anticoagulants in the post-DOAC era were older than their counterpart cohort from the pre-DOAC era $(\mathrm{p}<0.01)$. Similarly, patients who received an antiplatelet therapy or no treatment in the post-DOAC era had higher CCI for comorbidities ( $\mathrm{p}<0.05$ and $\mathrm{p}<0.01$, respectively), $\mathrm{CHA}_{2} \mathrm{DS}_{2}-\mathrm{VASc}$ and HAS-BLED scores compared with equivalent patients in the pre-DOACs era (all $p<0.01$ ) (Table 3). In light of the fact that other anticoagulants are usually prescribed for a brief period after hospital discharge, we have also considered the switch within the last 3 months of the follow-up period to other treatments.

In the pre-DOACs cohort, switch rates were 11.6, 7.3 and 66.1 in patients treated with VKA, antiplatelet or other anticoagulant agents, respectively (Figure 2A); interruptions were 9.7, 10.0 and 20.8, respectively. In the post-DOACs cohort, switch rates were 15.5, 5.9, 9.7 and 58.1 in patients treated with VKA, DOACs, antiplatelet or other anticoagulant agents, respectively (Figure $2 \mathrm{~A}$ ); interruptions were 11.3, 6.3, 14.6 and 23.5, respectively. In particular, in the pre-DOACs cohort, $34.2 \%$ of patients on other antithrombotics switched to VKA and $31.9 \%$ to an antiplatelet, whereas $9.8 \%$ of patients on VKA switched to an antiplatelet and $1.8 \%$ to other antithrombotics. 


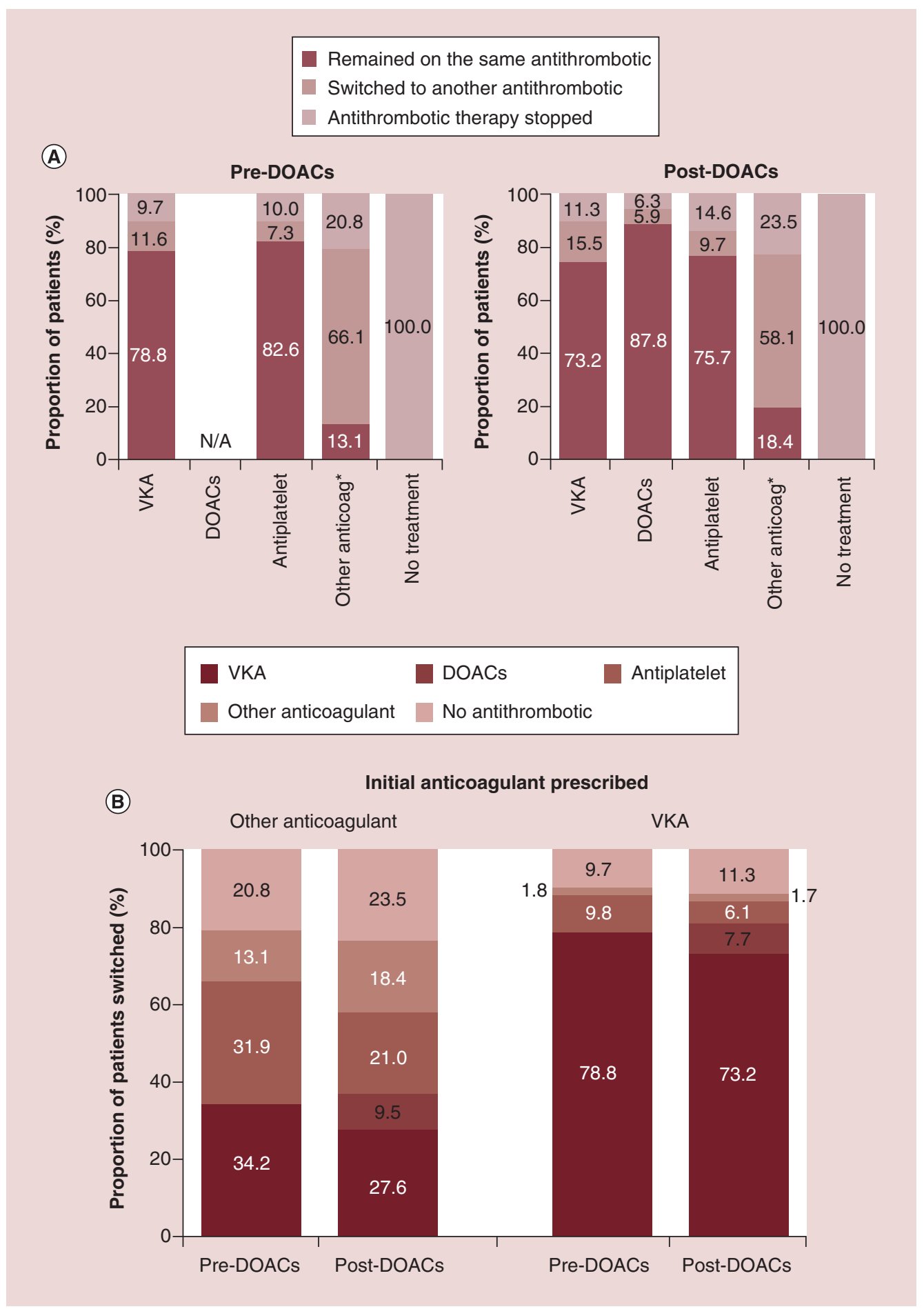

Figure 2. Antithrombotic use and treatment changes among patients with nonvalvular atrial fibrillation eligible for oral anticoagulants as per European Society of Cardiology guidelines during the 1-year follow-up in the pre- and post-direct oral anticoagulant cohorts. (A) Antithrombotic use and (B) details of antithrombotic treatment changes among patients with nonvalvular atrial fibrillation eligible for oral anticoagulants as per European Society of Cardiology guidelines during the 1-year follow-up in the pre- and post-direct oral anticoagulant cohorts. DOAC: Direct oral anticoagulant; Other anticoag: Other anticoagulant treatment; VKA: Vitamin K antagonist. 
Table 3. Demographic and baseline clinical characteristics and risk scores of patients with nonvalvular atrial fibrillation enrolled in 2010 and 2014, eligible for oral anticoagulants as per European Society of Cardiology guidelines, according to treatment.

\begin{tabular}{|c|c|c|c|c|c|}
\hline Pre-DOACs cohort & VKA & DOACs & Antiplatelet & Other anticoag & No treatment \\
\hline $\mathrm{N}$ & 2486 & / & 2348 & 789 & 955 \\
\hline Age, mean \pm SD & $74.9 \pm 8.6$ & / & $78.5 \pm 9.2$ & $78.0 \pm 9.3$ & $79.5 \pm 10.1$ \\
\hline Male, N (\%) & $1214(49)$ & / & $1014(43)$ & $336(43)$ & 358 (37) \\
\hline $\begin{array}{l}\text { Charlson Comorbidity } \\
\text { Index, mean } \pm \text { SD }\end{array}$ & $1.6 \pm 1.3$ & / & $1.8 \pm 1.5$ & $1.9 \pm 1.6$ & $1.1 \pm 1.4$ \\
\hline $\begin{array}{l}\text { Comorbidities of the } \\
\text { kidney and urinary tract, } \\
\mathrm{N}(\%)\end{array}$ & $41(1.6)$ & / & $43(1.8)$ & $30(3.8)$ & $18(1.9)$ \\
\hline $\begin{array}{l}\text { Comorbidities of the } \\
\text { hepatobiliary system and } \\
\text { pancreas, } \mathrm{N}(\%)\end{array}$ & $26(1.0)$ & / & $44(1.9)$ & $45(5.7)$ & $29(3.0)$ \\
\hline $\begin{array}{l}\mathrm{CHA}_{2} \mathrm{DS}_{2} \text {-VASc score, } \\
\text { mean } \pm \mathrm{SD}\end{array}$ & $3.8 \pm 1.2$ & / & $3.9 \pm 1.3$ & $4.0 \pm 1.3$ & $3.5 \pm 1.3$ \\
\hline $\begin{array}{l}\text { HAS-BLED score, } \\
\text { mean } \pm \text { SD }\end{array}$ & $2.4 \pm 0.9$ & / & $2.6 \pm 0.9$ & $2.7 \pm 1.0$ & $2.0 \pm 1.1$ \\
\hline $\mathrm{N}$ & 2337 & 671 & 1659 & 1108 & 827 \\
\hline Age, mean $\pm S D$ & $76.6 \pm 8.7^{\ddagger}$ & $77.5 \pm 8.4$ & $80.2 \pm 9.6^{\ddagger}$ & $79.2 \pm 9.5^{\ddagger}$ & $79.7 \pm 10.6$ \\
\hline Male, N (\%) & $1157(50)$ & $293(44)$ & $706(43)$ & $491(44)$ & $332(40)$ \\
\hline $\begin{array}{l}\text { Charlson Comorbidity } \\
\text { Index, mean } \pm \text { SD }\end{array}$ & $1.7 \pm 1.3^{\ddagger}$ & $1.6 \pm 1.4$ & $1.9 \pm 1.5^{\dagger}$ & $1.8 \pm 1.5$ & $1.5 \pm 1.4^{\ddagger}$ \\
\hline $\begin{array}{l}\text { Comorbidities of the } \\
\text { kidney and urinary tract, } \\
\mathrm{N}(\%)\end{array}$ & $37(1.6)$ & $5(0.7)$ & $38(2.3)$ & $47(4.2)$ & $27(3.3)$ \\
\hline $\begin{array}{l}\text { Comorbidities of the } \\
\text { hepatobiliary system and } \\
\text { pancreas, } N(\%)\end{array}$ & $14(0.6)$ & l & $22(1.3)$ & $46(4.2)$ & $19(2.3)$ \\
\hline $\begin{array}{l}\mathrm{CHA}_{2} \mathrm{DS}_{2} \text {-VASc score, } \\
\text { mean } \pm \mathrm{SD}\end{array}$ & $3.8 \pm 1.2$ & $3.9 \pm 1.1$ & $4.1 \pm 1.2^{\ddagger}$ & $4.0 \pm 1.2$ & $3.8 \pm 1.2^{\ddagger}$ \\
\hline $\begin{array}{l}\text { HAS-BLED score, } \\
\text { mean } \pm \text { SD }\end{array}$ & $2.5 \pm 0.8^{\ddagger}$ & $2.6 \pm 0.8$ & $2.8 \pm 0.8^{\ddagger}$ & $2.7 \pm 0.9$ & $2.5 \pm 1.0^{\ddagger}$ \\
\hline \multicolumn{6}{|c|}{$\begin{array}{l}{ }^{\dagger} p<0.05 \text { (pre-DOACs vs post-DOACs for each single group). } \\
{ }_{\ddagger} p<0.01 \text { (pre-DOACs vs post-DOACs for each single group). } \\
\text { DOAC: Direct oral anticoagulant; Other anticoag: Other antico }\end{array}$} \\
\hline
\end{tabular}

In the post-DOACs cohort, $27.6 \%$ of patients on other antithrombotics switched to VKA, 9.5\% to DOACs and $21.0 \%$ to an antiplatelet, whereas $7.7 \%$ of patients on VKA switched to DOACs, $6.1 \%$ to an antiplatelet and $1.7 \%$ to other antithrombotics (Figure 2B).

The most frequent MDC for the index hospitalization was the circulatory system in two-thirds of patients in both the pre- and post-DOACs cohort; in $\sim 10 \%$ of patients, the MDC was the nervous and respiratory system; other MDCs were found in $<5 \%$ of patients. Both in the pre- and post-DOACs cohort, $\sim 70 \%$ of patients had an index hospitalization stay of $<10$ days; NVAF was recorded as the main diagnosis in approximately one-third of patients, whereas in all other cases, NVAF was reported as the secondary diagnosis. Patients on heparin had treatment duration of 101 days for the pre-DOACs cohort and 119 days for the post-DOACs cohort.

\section{Discussion}

The purpose of this study was to evaluate treatment patterns in Italian patients with a discharge diagnosis of NVAF, who should receive anticoagulant treatment as per ESC guidelines current at the time of pre- and post-DOACs approval [19,20], and to assess patients' characteristics (in terms of age and comorbidity index) and risks (based on $\mathrm{CHA}_{2} \mathrm{DS}_{2}$-VASc and HAS-BLED scores).

In this real-world assessment, in both the considered periods, approximately $14 \%$ of patients discharged with a diagnosis of NVAF, eligible for OAC treatment according to the ESC guidelines [19], received no treatment upon hospital discharge. The second main finding was that in the pre- and post-DOACs periods the proportion of patients treated with VKA and other anticoagulants (no DOACs) were largely similar, whereas the proportion of 
patients treated with antiplatelets decreased from 36 to $25 \%$. This difference was largely because of the introduction of DOACs, with $10 \%$ of patients in the 2014 cohort being prescribed a DOAC (vs $0 \%$ in the 2010 cohort). The findings also showed that the proportion of patients discharged with a diagnosis of NVAF and who did not receive OACs or antiplatelet therapy decreased from 2010 to 2014.

These trends coincided with the 2012 update to the ESC guidelines for the management of AF [20]. Whereas aspirin was endorsed for patients at intermediate risk of stroke according to $\mathrm{CHADS}_{2}$ risk stratification in 2010, the 2012 update recommended that antiplatelets were to be considered only when a patient would refuse any OAC. Furthermore, in the 2012 update DOACs were for the first time an option for patients unable to receive a VKA $[19,20]$.

Overall, in the post-DOACs cohort of this study, $45 \%$ of patients were discharged with OAC (i.e., VKA or DOAC) therapy for the prevention of thromboembolism in line with the 2012 ESC recommendations [20]. Despite progress toward increased use of evidence-based OAC therapy, an unmet medical need remained for the $55 \%$ of patients who received no treatment, antiplatelet therapy or other anticoagulants not recommended by the ESC guidelines.

Our results are consistent with previously published studies from different populations, which demonstrated an increased use of OAC therapy and uptake of DOAC therapy in recent years $[7,9,11,12,21,22]$. However, the level of OAC underuse reported by Global and European Registries in the post-DOACs period (from 14 to $<30 \%)[7,9,10]$ is much lower than that reported in the present study $(55 \%)$. We should, however, emphasize that we have only considered patients observed retrospectively in the real-world setting; for this reason, our percentage of undertreated patients could be different from the registry data because of the different study setting and selection process [23]. Indeed, the reported underuse of OACs is higher in other studies in the post-DOACs era in Europe. For example, studies in the UK and Turkey report an $\sim 70 \%$ use of OAC therapy in patients diagnosed with AF after 2012 [11,12]. The latter study also reported a slightly higher use of DOAC versus VKA therapy in 2015 (35 and $37 \%$, respectively) [12], which is supported by analysis of data from the 2014 to 2015 cohort of the GARFIELD-AF registry (35 and 37\%, respectively) and a large DB analysis in Norway [22].

In line with the present analysis, the PREFER in AF registry reported that use of OACs appears to be especially low in Italy [14]. In this analysis, only 65\% of patients enrolled in Italy received an OAC at the 1-year follow-up, compared with $86 \%$ in the overall study population [9,14], suggesting that Italy may be lagging behind its European counterparts in the uptake of DOACs for NVAF. Notably, the use of DOACs in this analysis at the 1-year follow-up was much lower than the European mean (3.3 and 12.6\%, respectively) [14].

We observed that the availability of DOACs may have improved the proportion of patients treated with OACs: in fact, DOACs tended to be prescribed in patients previously treated with antiplatelet drugs. However, an increase in the use of 'other anticoagulants' from 12\% in 2010 to $17 \%$ in 2014 was also observed in our study, despite the 2012 ESC guidelines only endorsing the use of VKAs and DOACs (and antiplatelet therapy in cases where a patient refuses an OAC). In addition, our findings show that heparin users had a treatment duration of more than 100 days in the post-DOACs cohort. The results of our study are similar with a recent report showing an increase in the use of heparin products in patients with a diagnosis of DVT [24]. The 2015 national report on medicine use in Italy showed that $84.4 \%$ of DVT patients were treated with heparin or fondaparinux for more than 10 days [25]. These data were retrieved by the Nationwide OsMed Health-DB Database and have been validated by AIFA to describe drug consumption nationwide. Our data show differences between clinical practice and guidelines, we suggest that the use of DOACs could have modified these unappropriated prescriptions. In this real-world assessment, we also found that the average ages of patients with NVAF enrolled in this analysis was higher in the post-DOACs cohort compared with the pre-DOACs cohort (78.4 and 77.2 years, respectively; $\mathrm{p}<0.001$ ). In the post-DOACs cohort, among DOACs users the mean age was 77.5 years; this cohort of patients was slightly older than the VKA users (76.6 years), but younger than patients who received antiplatelet, other anticoagulant therapy or no treatment. Furthermore, patients prescribed a DOAC in 2014 had lower $\mathrm{CHA}_{2} \mathrm{DS}_{2}$-VASc and HAS-BLED scores compared with the other patient groups. Indeed, physicians may have preferred to prescribe DOACs to lower-risk patients in the period after the introduction of DOACs in Italy, increasing the average mean age and risk scores of patients in the other treatment categories.

Many factors play a crucial role in the underuse of OACs, including patient characteristics (i.e., older age or female sex), comorbidities (i.e., hepatic or renal disease), non-compliance or low balance between therapeutic efficacy and safety risks associated with drugs [26-28]. In addition, fear of treatment and lack of access to the healthcare system may contribute to underuse [27]. Specific to Italy, uptake of DOACs might be lagging behind other countries in 
Europe because reimbursement was not possible until mid-2013 for dabigatran, late 2013 for rivaroxaban and early 2014 for apixaban. Furthermore, Renda et al. noted that VKA therapy was better managed in Italy compared with other European countries, with higher time in the therapeutic range reported; this may have lessened the incentive to use the newer DOAC therapies. However, patient-reported treatment satisfaction with VKA therapy was low [14]. Analysis of OAC use over the past few years in Italy and across Europe would be informative to gauge any further changes in clinical practice with the release of the present 2016 update of the ESC guidelines for the management of $\mathrm{AF}[5]$ and increased experience with the DOACs.

\section{Limitations}

Our results should be interpreted in the context of the limitations of this study. Our cohort of patients reflected real clinical practice, and the results must be interpreted, taking into account of limitations related to the observational nature of the study, based on data collected through administrative and laboratory DBs. Furthermore, the cohorts consisted of patients discharged from hospital and, therefore, did not include patients diagnosed and managed by a general practitioner. Because the information concerning the comorbidities and the information relative to the severity of the pathology is not available, a proxy of the comorbidities was used; this criterion can lead to a bias relative to the actual prevalence of the comorbidities (possible underestimation). The results and conclusions of this study are limited to the population analyzed.

\section{Conclusion}

The results of this study suggest some improvement in adherence to clinical recommendations in Italy in line with findings from other European and Global datasets. However, an unmet medical need remains among patients with NVAF. The present findings suggest that a proportion of patients are still undertreated or do not receive appropriate therapy for NVAF management. Considering these results, educational interventions and drug therapy management programs, may help to increase the use of OAC therapies in the appropriate patients.

\section{Future perspective}

The principal aim of this study was to capture real-world data on patient management and outcomes from routine clinical practice in the field of stroke prevention in patients with NVAF in Italy. Previously published analyses of data from registries have identified substantial gaps between antithrombotic prescribing in the real world and guideline recommendations. Although anticoagulation provides a net clinical benefit in patients with $\mathrm{AF}$, there is increasing evidence to suggest that the majority of patients not treated with an OAC are actually eligible for treatment. Antithrombotic prescribing patterns have evolved over the past few years, with DOACs becoming the most prescribed class of agents, while the use of VKAs is slowly diminishing; a trend that is anticipated to continue over the coming years. Cumulatively, these analyses will provide a comprehensive overview of patients with NVAF and their management, as well as helping to improve patient outcomes.

\section{Summary points}

- A substantial proportion of patients (approximately $14 \%$ ) who were discharged with a diagnosis of nonvalvular atrial fibrillation and eligible for oral anticoagulant (OAC) treatment remained untreated.

- In the pre- and post-direct OAC (DOAC) cohorts, the proportion of patients treated with vitamin $\mathrm{K}$ antagonists and other anticoagulants (not DOACs) was largely similar.

- The proportion of patients treated with antiplatelets decreased following the introduction of DOACs from $36 \%$ in 2010 to $25 \%$ in 2014 .

- Overall, the proportion of patients discharged with a diagnosis of nonvalvular atrial fibrillation who were eligible for OAC but did not receive OACs or antiplatelet therapy decreased from 62\% in 2010 to 55\% in 2014.

- Despite this improvement in adherence to clinical guidelines, an unmet medical need remained for the $55 \%$ of patients who received no treatment, antiplatelet therapy or other anticoagulants not recommended by the European Society of Cardiology guidelines. 
Financial \& competing interests disclosure

J-B Briere and K Bowrin are employees of Bayer AG. Manuscript development was supported by funding from Bayer. The other authors have no conflicts of interest to declare. The authors have no other relevant affiliations or financial involvement with any organization or entity with a financial interest in or financial conflict with the subject matter or materials discussed in the manuscript apart from those disclosed.

Editorial assistance was utilized in the production of this manuscript with funding from Bayer AG.

\section{Ethical conduct of research}

The authors state that they have obtained appropriate institutional review board approval or have followed the principles outlined in the Declaration of Helsinki for all human or animal experimental investigations. In addition, for investigations involving human subjects, informed consent has been obtained from the participants involved.

\section{Open access}

This work is licensed under the Attribution-NonCommercial-NoDerivatives 4.0 Unported License. To view a copy of this license, visit http://creativecommons.org/licenses/by-nc-nd/4.0/

\section{References}

1. Zoni-Berisso M, Filippi A, Landolina $\mathrm{M}$ et al. Frequency, patient characteristics, treatment strategies, and resource usage of atrial fibrillation (from the Italian Survey of Atrial Fibrillation Management [ISAF] study). Am. J. Cardiol. 111(5), 705-711 (2013).

2. Ermini G, Perrone V, Veronesi C, Degli Esposti L, Di Pasquale G. Antithrombotic prophylaxis of atrial fibrillation in an Italian real-world setting: a retrospective study. Vasc. Health Risk Manag. 13, 239-246 (2017).

3. Ceornodolea AD, Bal R, Severens JL. Epidemiology and management of atrial fibrillation and stroke: review of data from four European countries. Stroke Res. Treat. 2017, 8593207 (2017).

4. Centers for Disease Control and Prevention. Atrial fibrillation fact sheet (2017). www.cdc.gov/dhdsp/data_statistics/fact_sheets/fs_atrial_fibrillation.htm

5. Kirchhof P, Benussi S, Kotecha D et al. 2016 ESC Guidelines for the management of atrial fibrillation developed in collaboration with EACTS. Eur. Heart J. 37(38), 2893-2962 (2016).

6. Connolly SJ, Ezekowitz MD, Yusuf S et al. Dabigatran versus warfarin in patients with atrial fibrillation. N. Engl. J. Med. 361(12), 1139-1151 (2009).

7. Huisman MV, Rothman KJ, Paquette $\mathrm{M}$ et al. The changing landscape for stroke prevention in AF: findings from the GLORIA-AF registry phase 2. J. Am. Coll. Cardiol. 69(7), 777-785 (2017).

8. De Caterina R, Ammentorp B, Darius $\mathrm{H}$ et al. Frequent and possibly inappropriate use of combination therapy with an oral anticoagulant and antiplatelet agents in patients with atrial fibrillation in Europe. Heart 100(20), 1625-1635 (2014).

9. Kirchhof P, Ammentorp B, Darius H et al. Management of atrial fibrillation in seven European countries after the publication of the 2010 ESC Guidelines on atrial fibrillation: primary results of the PREvention oF thromboemolic events-European Registry in Atrial Fibrillation (PREFER in AF). Europace 16(1), 6-14 (2014).

10. Camm AJ, Accetta G, Al Mahmeed W et al. Impact of gender on event rates at 1 year in patients with newly diagnosed non-valvular atrial fibrillation: contemporary perspective from the GARFIELD-AF registry. BMJ Open 7(3), e014579 (2017).

11. Durham TA, Hassmiller Lich K, Viera AJ et al. Utilization of standard and target-specific oral anticoagulants among adults in the United Kingdom with incident atrial fibrillation. Am. J. Cardiol. 120(10), 1820-1829 (2017).

12. Basaran O, Beton O, Dogan V et al. ReAl-life Multicenter Survey Evaluating Stroke prevention strategies in non-valvular atrial fibrillation (RAMSES study). Anatol. J. Cardiol. 16(10), 734-741 (2016).

13. Deambrosis P, Bettiol A, Bolcato J et al. Real-practice thromboprophylaxis in atrial fibrillation. Acta Pharm. 67(2), $227-236$ (2017).

14. Renda G, Patti G, Sangiuolo R et al. Management of thromboembolic risk in patients with atrial fibrillation in Italy: follow-up data from the PREFER in AF European Registry. G. Ital. Cardiol. (Rome) 17(11), 922-931 (2016).

15. Perrone V, Saragoni S, Buda S, Broccoli A, Degli Esposti L. Pharmacoutilization of epoetins in naive patients with hematological malignancies in an unselected Italian population under clinical practice setting: a comparative analysis between originator and biosimilars. Biologics 10, 157-165 (2016).

16. Agenzia Italiana del Farmaco (AIFA). Guideline for the classification and conduction of the observational studies on medicines (2010). www.agenziafarmaco.gov.it/ricclin/sites/default/files/files_wysiwyg/files/CIRCULARS/Circular\%2031st\%20May\%202010.pdf

17. Farner S, Malkani A, Lau E, Day J, Ochoa J, Ong K. Outcomes and cost of care for patients with distal radius fractures. Orthopedics 37(10), e866-e878 (2014).

18. Charlson ME, Pompei P, Ales KL, MacKenzie CR. A new method of classifying prognostic comorbidity in longitudinal studies: development and validation. J. Chronic Dis. 40(5), 373-383 (1987). 
19. Camm AJ, Kirchhof P, Lip GYH et al. Guidelines for the management of atrial fibrillation: the task force for the management of atrial fibrillation of the European Society of Cardiology (ESC). Eur. Heart J. 31(19), 2369-2429 (2010).

20. Camm AJ, Lip GYH, De Caterina R et al. 2012 focused update of the ESC Guidelines for the management of atrial fibrillation: an update of the 2010 ESC Guidelines for the management of atrial fibrillation. Developed with the special contribution of the European Heart Rhythm Association. Eur. Heart J. 33(21), 2719-2747 (2012).

21. Camm AJ, Accetta G, Ambrosio G et al. Evolving antithrombotic treatment patterns for patients with newly diagnosed atrial fibrillation. Heart 103, 307-314 (2017).

22. Urbaniak AM, Strom BO, Krontveit R, Svanqvist KH. Prescription patterns of non-vitamin K oral anticoagulants across indications and factors associated with their increased prescribing in atrial fibrillation between 2012-2015: a study from the Norwegian prescription database. Drugs Aging 34(8), 635-645 (2017).

23. Fox KAA, Accetta G, Pieper KS et al. Why are outcomes different for registry patients enrolled prospectively and retrospectively? Insights from the global anticoagulant registry in the FIELD-Atrial Fibrillation (GARFIELD-AF). Eur. Heart J. Qual. Care Clin. Outcomes 4(1), 27-35 (2018).

24. Zhou A, Winkler A, Emamifar A et al. Is the incidence of heparin-induced thrombocytopenia affected by the increased use of heparin for VTE prophylaxis? Chest 142(5), 1175-1178 (2012).

25. Agenzia Italiana del Farmaco (AIFA). L'uso dei farmaci in Italia—rapporto OsMed (2015). www.aifa.gov.it/sites/default/files/Rapporto_OsMed_2015_AIFA-acc.pdf

26. Wehbe RM, Yadlapati A. Underuse of oral anticoagulants for nonvalvular atrial fibrillation: past, present, and future. Tex. Heart Inst. J. 43(4), 287-290 (2016).

27. Gattellari M, Worthington J, Zwar N, Middleton S. Barriers to the use of anticoagulation for nonvalvular atrial fibrillation: a representative survey of Australian family physicians. Stroke 39(1), 227-230 (2008).

28. Agarwal S, Bennett D, Smith DJ. Predictors of warfarin use in atrial fibrillation patients in the inpatient setting. Am. J. Cardiovasc. Drugs 10(1), 37-48 (2010). 